\title{
Bid Increment Adjusting Strategies in English Auction-based Sponsored Search
}

\author{
Chen-Kun Tsung ${ }^{1, *}$, Hann-Jang Ho ${ }^{2}$ and Sing-Ling Lee ${ }^{1}$ \\ ${ }^{1}$ Institute of Computer Science and Information Engineering, National Chung Cheng University, No.168, Sec. 1, University Rd., Min- \\ Hsiung Township, Chia-yi County 62102, Taiwan (R.O.C.) \\ ${ }^{2}$ Department of Applied Digital Media, WuFeng University, No. 117, Sec. 2, Chiankuo Rd., Min-Hsiung Township, Chia-yi County \\ 62153, Taiwan (R.O.C.)
}

Received: 26 Jun. 2013, Revised: 31 Oct. 2013, Accepted: 1 Nov. 2013

Published online: 1 May. 2014

\begin{abstract}
English auctions are applied in many current e-commerce applications because of the easy implementation. Some sponsored search advertising (SSA) services consider the ascending bidding rules of English auctions to determine winners. However, the high computation cost of determining winners is the major drawback of the English auction-based SSAs. Because the SSA is an online service, Internet users do not wait for a long time to receive the search result pages. In this paper, we provide a mechanism to reduce the overhead of computing winners and maximize the revenue obtained by the search engine provider (SEP). Our proposed mechanism adopts the minimum increase price (MIP), which is similar to the central price in the English auction, to indicate how much should be raised for the bids with updated prices. We design a uniform MIP adjusting strategy and an adaptive MIP adjusting strategy to determine the MIP setting in each round. We analyze the convergent speed of two proposed MIP adjusting strategies. According to our simulation results, we conclude the following properties. For all the SEP revenue considerations, the adaptive MIP adjusting strategy is outstanding in popular keywords, and rare keywords for the uniform MIP adjusting strategy. Opposite results are obtained for the convergent speed.
\end{abstract}

Keywords: Sponsored Search Advertising, English Auction, Generalized Second Price Auction, Convergent Speed, AdditiveIncrease/Multiplicative-Decrease

\section{Introduction}

The utilization of the search service is continuously increased since the Internet became popular. Most search engine providers (SEPs) use sponsored search advertising (SSA) services to recommend potential advertisements for Internet users $[4,2,6]$. The SSAs combine the online advertising with the search service. When Internet users issue keyword queries, the advertisements corresponding to the received keyword are posted on the screen. Every time the SSA receives a keyword query, we treat it as a round. Because all advertisements are related to the queried keywords, most Internet users favor the advertisements recommended by SSAs. Therefore, SSAs bring major incoming for SEPs [4, 12].

The charging functions of SSAs involve the pay-per-click structure and the generalized second price (GSP) principle [4]. Under the pay-per-click structure, each advertiser pays for advertising only when the Internet user clicks their advertisement. Simultaneously, the advertiser gains a private worth from the click, and we call the worth as the valuation. GSP charges the essential price of winning a slot, i.e., the bid price proposed by the next-ranked advertiser.

Because SSAs are online services, easy implementation is necessary for designing mechanisms. The generalized English auction [4] and the ascending implementation [5] adopt the full process of the English auction [13] to rank advertisers. Both mechanisms consider a central price which is increased automatically. Every time the central price is increased, advertisers must determine to accept the price to stay in the auction, or reject the price and drop out. Eventually, the last dropped advertiser wins the best slot, the second-to-last dropped advertiser wins the second slot, etc.

\footnotetext{
*Corresponding author e-mail: tck95p@gmail.com
} 
The English auction is a well known mechanism, so advertisers know how to bid to achieve the objective in the English auction-based SSAs. However, the time consumption may be an implementation issue as the winners are computed via the full process of English auction. Considering that some advertisers' valuations are very close. The increment of the central price must be sufficient low to identify the ranks of these advertisers. Thus, computing a ranking result requires a long process when the valuations are high. Internet users do not wait for a long time to receive a search result page. So, decreasing the overhead of calculating winners in each round for English auction-based SSAs is our first goal.

The central price determines the final bid values. This implies the SEP revenue depends on the central price. However, determining the central price is a case-by-case study [19]. Our second objective is to design a strategy to compute the central price to maximize the SEP revenue.

In this paper, we proposed a mechanism, termed the non-decreasing sponsored search auction (NDSSA), to realize the following objectives. 1) Minimizing the overhead of computing the auction result in each round, and 2) maximizing the SEP revenue. To achieve the first objective, we extend the bidding rule of the English auction from ascending to non-decreasing. The non-decreasing bidding rule indicates that the advertisers can submit the same bid values as that proposed in the last round or higher prices rather than the continuously increased values. Then, the non-decreasing bidding rule is used to increase the bidding competition throughout the auction rather than to determine the ranking result in each round. The NDSSA adopts the rank-by-bid principle to determine winners. Therefore, the overhead of determining winners in each round can be reduced.

To realize our second goal (maximizing the SEP revenue), we replace the central price of the English auction by the minimum increase price (MIP). The MIP indicates the minimum bid increment for the bids with updated prices. If the advertiser proposes a new price, the raised amount must be higher than the MIP.

The hardness of determining the MIP in the real world SSAs is that the valuations are unreachable. Thus, it is hard to compute optimal MIP settings that the derived bid prices are close to the valuations. In this paper, we design a uniform and an adaptive MIP adjusting strategies to compute the MIP. The uniform strategy, we termed as the fixed-MIP, utilizes an invariant MIP throughout the auction. So the SEP revenue in the fixed-MIP depends on the initial MIP settings. The adaptive strategy which is named as the AIMD-MIP applies the idea of additive-increase/multiplicative-decrease [9] to compute the MIP in each round. The AIMD-MIP compares the bid profiles received in two successive rounds. If no bid is updated, this implies that the MIP is too high to raise bids for advertisers, the AIMD-MIP reduces the MIP to one half of the current MIP value. Otherwise, the MIP is increased by one to raise the bidding competition.
We evaluate the convergent speed and the SEP revenue for the NDSSA. The convergent speed indicates the number of rounds that the NDSSA requires to meet the stable outcome. In the stable outcome, each advertiser bids at their highest price, and no bid will be updated in the future. We first compute the convergent speed for the fixed-MIP and AIMD-MIP, and analyze the essential condition that the fixed-MIP converges faster than the AIMD-MIP. In our simulation, we evaluate the convergent speed and four metrics regarding SEP revenue for the fixed-MIP and AIMD-MIP with various initial MIP settings. We conclude the following properties.

1.There is a tradeoff between maximizing the SEP revenue and minimizing the convergent speed.

2.For optimizing the convergent speed, the AIMD-MIP is suitable for rare keywords, and the fixed-MIP with appropriate initial MIP is proper for popular keywords.

3.For maximizing the SEP revenue, the AIMD-MIP is outstanding for popular keywords; the fixed-MIP is distinguished for rare keywords.

\section{Related Works}

There are two English auction-based SSA mechanisms: the generalized English auction proposed by Edelman et al. [4] and the ascending implementation proposed by Babaioff and Roughgarden [5]. The generalized English auction focus on the assumption that the valuations are common knowledge while this restriction is not considered in the ascending implementation. Both mechanisms derived that the SEP gains the same revenue as that obtained in the Vickrey-Clarke-Groves (VCG) auction [11].

Because both mechanisms utilize the full process of English auction to compute winners, determining winners may require high computation cost. To reduce the cost of computing winners, the NDSSA adopts the rank-by-bid principle and increases advertisers' bid prices round-by-round. Thus, the overhead of computing winners in the NDSSA is less than that in the generalized English auction and the ascending implementation.

The central price can be considered as a range which is called as the bid levels $[14,15,16,17]$. In the beginning, the auctioneer announces the first bid level and checks bidders' willingness of paying in this range. Then, the auction announces the second bid level which is higher than the first one. As increasing the bid levels, bidders' bid prices are increased. Eventually, the bidder stays in the highest bid level wins the auction item.

Zhang et al. proposed a greedy approach to determine the bid level [14] and showed their approach is robust for capturing advertisers' valuations. Rothkopf and Harstad focused on computing the number of bid levels [17]. Rogers et al. [15] and David et al. [16] proposed the approaches to maximize the auctioneer's revenue. Although the bid level reduces the computation cost of 
determining winners, advertisers with higher valuations may not win the better slot if valuations are very close.

Combining the advantages of the central price and the bid level, real world English auctions utilize the range bid increment. For example, according to the bidding rule of the Taiwan Yahoo auction ${ }^{1}$, the bid increment is $\$ 10$ within the prices $\$ 0$ and $\$ 499$, the bid increment is $\$ 30$ within the prices $\$ 500$ and $\$ 1999$, etc. However, if applying the range bid increment to the English auction-based SSAs, the advertisers with higher valuations may not obtain better slots. Because the bid increments are large in high bid prices due to the convergent speed consideration, advertisers with higher valuations may win the slots which is worse than that obtained by the advertisers with lower valuations. Our adaptive MIP adjusting strategy dynamically computes the MIP setting in each round, therefore, the problem resulted from the range bid increment can be resolved in the NDSSA.

\section{Auction Model}

Consider a repeated SSA with $N$ advertisers $A D=\left\{a d_{1}, a d_{2}, \ldots, a d_{N}\right\}$ and $K$ slots $S L=\left\{s l_{1}, s l_{2}, \ldots, s l_{K}\right\}$, where $N>K$. The repeated model focuses on a given keyword phrase. When a keyword query arrives, the SSA recommends advertisements by the following steps.

Step 1. Collect the bid profile $B=\left\{b_{1}, b_{2}, \ldots, b_{N}\right\}$. The SSA requests each advertiser $a d_{i}$ to propose a bid value $b_{i}$ to specify the maximum price that $a d_{i}$ is willing to pay for advertising.

Step 2. Determine winners. The SSA ranks advertisers based on a predefined ranking function.

Step 3. Compute payments. The SSA charges each winner by a given charging function.

Step 4. Output the auction result. The SSA announces the ranking result and payment profile to all advertisers.

Each slot $s l_{j}$ has a click-through-rate (CTR) $\theta_{j}$ to indicate the click probability that Internet users click the advertisements on $s l_{j}$. We consider the CTR as the decreasing distribution, i.e., $\theta_{1}>\theta_{2}>\ldots \theta_{k}$; that is, the advertising slot rearranged in a higher position receives more clicks than that in a lower position.

When an Internet user clicks the advertisement of $a d_{i}$, $a d_{i}$ pays the GSP price $p_{i}=b_{i+1}$, and gains the valuation $v_{i}$. We consider that each $a d_{i}$ is conservative; that is, the bid value is bounded by the valuation, i.e., $b_{i} \leq v_{i}$. For convenience, we assume that $a d_{i}$ wins $s l_{i}$. Given a bid profile $B$, the utility of $a d_{i}$ is $u_{i}(B)=\left(v_{i}-p_{i}\right) \theta_{j}=\left(v_{i}-b_{i+1}\right) \theta_{j}$, according to the charging rule of GSP.

Many bidding strategies are proposed to formulate real world bidding behaviors, such as forward-looking

1 http://help.yahoo.com/kb/index?page=content\&id=SLN11175\&locale=zh_TW responses [2], balanced bidding strategies [1], and strategic bidding strategies [3]. Most bidding strategies emphasize the utility maximization for advertisers. So, we consider that all advertisers adopt utility-maximization strategies to determine bid values.

Given a bid profile $B, a d_{i}$ first computes the target slot $s l_{k}$ which has maximum utility, and then, calculates the bid price by applying Eq. (1), where $s l_{i}$ is the current obtained slot. If $s l_{k}$ is the current obtained slot, $a d_{i}$ will submit $b_{i}$ again, and otherwise $b_{k}+\varepsilon, \forall \varepsilon>0$ which is slightly higher than $b_{k}$.

$$
F(B)= \begin{cases}b_{i}, & \text { if } s l_{k}=s l_{i} \\ b_{k}+\varepsilon, & \text { otherwise. }\end{cases}
$$

The VCG auction is incentive-compatible [11]. The SEP revenue obtained in the VCG is the lower bound of some mechanisms $[1,2,4]$. VCG ranks advertisers to maximize the social welfare. This guarantees the advertisers with higher valuations obtain the slots with more clicks. The winner of $s l_{j}$ pays the difference between the social welfare when the winner joins and does not join the auction, and that is shown in Eq. (2).

$$
\sum_{j=s+1}^{K+1}\left(\theta_{j-1}-\theta_{j}\right) b_{j}
$$

\section{Solution}

\subsection{Non-decreasing Sponsored Search Auction}

The generalized English auction and the ascending implementation apply the full process of the English auction to determine winners. The computation cost of determining winners is high when some advertisers' valuations are close. Because the SEP can not access the valuations, the central price must be set as small. Thus, determining winners in each round requires high computation time. The NDSSA overcomes the high computation cost of determining winners while the SEP revenue is maximized.

We first extend the bidding rule of the English auction from ascending to non-decreasing. The non-decreasing bidding rule is used to increase the bidding competition round-by-round rather than to compute the winners. The winners are determined via the rank-by-bid strategy. Each advertiser can propose the bid which is either identical to that used in the last round or a higher price. Therefore, the overhead of computing winners is minimized.

The NDSSA considers the MIP to control the bidding competition between advertisers. The MIP is a threshold of proposing a new bid price. Because the non-decreasing bids are available, only the updated bids are subject to the MIP restriction. So, the SEP must determine two parameters before the NDSSA starts: the initial MIP and the MIP adjusting strategy which is used to compute the MIP in each round.

The procedure of the NDSSA is shown as follows. 
Definition 1(Non-decreasing Sponsored Search Auction). Given an MIP value $m$ and an MIP adjusting strategy MS. When a keyword query arrives, the NDSSA performs the following processes.

Step 1. Collect the bid profile $B=\left\{b_{1}, b_{2}, \ldots, b_{N}\right\}$.

Step 2. Verify the validity of each $b_{i}$ by $m$.

Step 3. Determine winners by the rank-by-bid strategy.

Step 4. Compute the new MIP by the given MS.

Step 5. Output the auction result.

In Step 2, feasible bid prices are shown in Eq. (3), where $b_{i}^{-}$is the bid price of $a d_{i}$ received in the last round, and $\forall \varepsilon \geq 0$. In Step 3, we assume that all bids have different prices, i.e., $b_{i} \neq b_{s}, \forall i \neq s$, so the tie-breaking mechanism is unnecessary. In Step 4, we propose two MIP adjusting strategies to determine the MIP in each round. In Step 5, the auction result includes the bid profile, payment profile, ranking result, and the new MIP.

$$
b_{i}= \begin{cases}b_{i}^{-}+m+\varepsilon, & \text { if } b_{i} \neq b_{i}^{-} \\ b_{i}^{-}, & \text {otherwise } .\end{cases}
$$

In the real world SSAs, each advertiser receives a daily advertising report. Advertisers can adjust the auction settings according to the advertising reports. Thus, we consider the NDSSA as a daily-reset mechanism. When the NDSSA restarts, the SEP can utilize another initial MIP or new MIP adjusting strategy while advertisers can submit any bid price without MIP restriction.

\subsection{Bid Increment Adjusting Strategies}

We propose a uniform MIP adjusting strategy with constant MIP termed fixed-MIP $f(m)$, and an adaptive MIP adjusting strategy labeled by AIMD-MIP $a(m)$. The MIP in the fixed-MIP is invariant, and dynamic for the AIMD-MIP.

Considering the initial MIP $m^{1}$, the MIP in the fixed-MIP is identical to $m^{1}$ throughout the auction, i.e., $f(m)=m^{1}$. After the SEP announces $m^{1}$, all advertisers obey the bidding rule of Eq. (3). The MIP is updated when the auction is restarted every day.

We utilize the concept of the additive-increase/multiplicative-decrease (AIMD) to design the AIMD-MIP. The AIMD is used to avoid network congestion, and the definition is shown as below.

\section{Definition 2}

(Additive-Increase/Multiplicative-Decrease [7]). The AIMD is an algorithm of TCP congestion avoidance. Given an unknown network bandwidth, the objective is to probe the bandwidth to maximize the packet transmission rate. AIMD specifies a threshold to indicate the maximum packet transmission rate. When no packet loses, which implies current packet transmission rate could be raised, the threshold is increased linearly. If packets are lost, which indicates the packet transmission rate is overestimated, the threshold is decreased exponentially.
Table 1: Event mapping for the TCP network and the NDSSA.

\begin{tabular}{|c|c|}
\hline Events in TCP network & Events in NDSSA \\
\hline \hline Packet transmission threshold & MIP value \\
\hline Congestion free & Any bid with updated price \\
\hline Network congestion & No bid with updated price \\
\hline
\end{tabular}

Table 1 shows the event mapping from the TCP network to the NDSSA. The MIP is increased by one if any advertiser updates the bid price. Otherwise, the MIP is decreased to a half of current value. The AIMD-MIP computes the MIP values according to Eq. (4). Given the current MIP $m, B$ and $B^{\prime}$ are the bid profiles received in the current round and the last round. If $B \neq B^{\prime}$, which implies the MIP is not sufficiently high, the AIMD-MIP outputs $m+1$, and $m / 2$ otherwise.

$$
a(m)=\left\{\begin{array}{l}
m+1, \text { if } B \neq B^{\prime}, \\
\lfloor m / 2\rfloor, \text { and otherwise. }
\end{array}\right.
$$

\section{Convergent Speed Analysis}

We confirm the convergency of the NDSSA before analyzing the convergent speed. Given an MIP adjusting strategy with arbitrary initial MIP, the NDSSA can produce stable outcomes.

Lemma 1.The NDSSA converges to a stable outcome when all advertisers are conservative.

Proof.The cycling bidding $[1,6]$ is eliminated in the NDSSA because the non-decreasing bidding rule is considered. Moreover, conservative advertisers do not overbid, i.e., $b_{i} \leq v_{i}$, so the bid prices are bounded by valuations. Each advertiser stops increasing bid prices when: 1) staying in the current slot is more beneficial than winning another slot, or 2) updating the bid price results in overbidding. Therefore, the stable outcome can be guaranteed.

We analyze the properties of the worst case for the convergent speed in Lemma 2. We define the available bid price $(\mathrm{ABP})$ as the price difference between the initial bid value and the valuation for an advertiser. The following discussions are under the assumption that all information is common knowledge. We use the stable bid prices to indicate the bid values in the stable outcomes.

Lemma 2. Given the current MIP value $m$, the worst case of convergent speed includes following properties.

1.The maximum ABP dominates the convergent speed.

2.For the advertiser $a d_{i}$ who has maximum $A B P$, we have $b_{i}>v_{i}-m$, where $b_{i}$ is the stable bid price of $a d_{i}$.

$3 . a d_{i}$ and another advertiser $a d_{x}$ compete for winning the same target slot $s l_{j}$. 
Proof. Suppose the advertiser $a d_{i}$ has the maximum ABP $\delta_{i}$. For the first property, because $a d_{i}$ has maximum ABP and all advertisers obey the same MIP, no advertiser can update the bid after $a d_{i}$ meets the maximum bid price. So, the convergent speed depends on $\delta_{i}$.

We assume that the second property is incorrect, and thus there exists a bid price $b_{i}^{*}$ results in the worse convergent speed then $b_{i}$. So, we have $b_{i}>v_{i}-m \geq b_{i}^{*}$. Because $b_{i} \geq b_{i}^{*}$, reaching $b_{i}^{*}$ does not require more rounds than reaching $b_{i}$. Thus, the assumption has a contradiction, and the second property is correct.

For the third property, if $s l_{j}$ is targeted by only $a d_{i}$, $a d_{i}$ may stop increasing bid price when obtaining $s l_{j}$. This implies $b_{i} \leq v_{i}-m$ where $b_{i}$ is the stable bid price of $a d_{i}$. This violates the second property, so more than one advertiser have the same target slot in the worst case.

The worst case in terms of the convergent speed is formulated in Definition 3.

Definition 3(Worst Case of Convergent Speed). Consider $a d_{i}$ has the maximum $A B P \delta_{i}$, and $a d_{i}$ and $a d_{x}$ have the same target slot $s l_{j}$. When $a d_{i}$ and $a d_{x}$ take turns at winning $s l_{j}$, the NDSSA requires most rounds to meet stable outcomes with $v_{i}<b_{i}+m$, where $b_{i}$ is the stable bid price of $a d_{i}$ and $m$ is the MIP applied in the stable outcome.

From [10], increasing the number of advertisers leads to small bid prices and slow convergent speed. However, several advertisers compete for one slot will not reduce the convergent speed in the NDSSA. Because the bid increment is bounded by the MIP, two advertisers compete for one target slot is sufficient to express the worst case of the convergent speed in the NDSSA. Hence, we can analyze the convergent speed of the fixed-MIP and the AIMD-MIP in the case shown in Definition 3.

\subsection{Fixed-MIP Strategy}

Theorem 1.Given the initial MIP $m^{1}$. Suppose ad has the maximum $A B P \delta_{i}$, the convergent speed of fixed-MIP is at most:

$$
r^{f}=2\left\lceil\frac{\delta_{i}}{m^{1}}\right\rceil
$$

Proof. We have $f(m)=m^{1}$, where $m$ is the current MIP. In Definition $3, a d_{i}$ requires at most $\left\lceil\delta_{i} / m^{1}\right\rceil$ rounds to meet the stable bid price. Furthermore, the worst case takes place when another advertiser has the same target slot as that of $a d_{i}$, and they take turns to update bid prices. Thus, the NDSSA requires double rounds to meet stable outcomes, and we have $r^{f}=2\left\lceil\delta_{i} / m^{1}\right\rceil$.

\subsection{AIMD-MIP Strategy}

The MIP adjustment process of the AIMD-MIP includes two phrases: the MIP increase phrase and the MIP

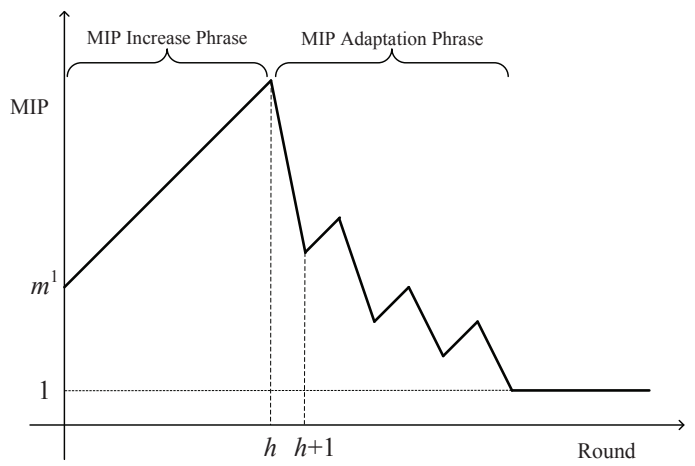

Fig. 1: The MIP modification process of the AIMD-MIP in the worst case.

adaptation phrase. The MIP is continuously raised in the MIP increase phrase. The couples of the MIP decrease and the MIP increase take place several times in the MIP adaptation phrase. When the MIP is decreased to one and no advertiser can update bid price, the NDSSA converges. Considering the MIP is decreased first in $(h+1)^{\text {th }}$ round, Figure 1 shows the MIP adjustment process of the AIMD-MIP.

We first prove that Figure 1 is the worst case regarding the convergent speed of the AIMD-MIP. We generalize the ABP definition and use $\delta_{i}^{s}$ to indicate the price gap between the bid value in $s^{\text {th }}$ round and the valuation. In other words, the labels $\delta_{i}^{1}$ and $\delta_{i}$ indicate the same value.

Lemma 3. Figure 1 shows the worst case of the convergent speed in the AIMD-MIP. In first $h$ rounds, the MIP is continuously increased. Then, the MIP variation couples with the pairs of the MIP decrease and MIP increase until the MIP is reduced to one.

Proof.Assume that the MIP adjustment process drawn in Figure 1 is not the worst case. So, there is a factitious case which results in worse convergent speed than that illustrated in Figure 1. Because the MIP is decreased in $(h+1)^{t h}$ round, the MIP adaptation phrase of the factitious case is longer than that shown in Figure 1. This indicates that the factitious case includes two or more successive MIP decreases or MIP increases.

If the factitious case suffers two successive MIP decreases, as shown in Figure 2(a), we prove that it is incorrect. Supposing the MIP is decreased in $s^{\text {th }}$ and $(s+1)^{t h}$ rounds in the factitious case. Because the MIP is decreased in $(s+1)^{t h}$ round, the remainder ABP in the factitious case is less than that in the case of Figure 1. Thus, the factitious case does not require more rounds to converge than the case drawn in Figure 1.

If the factitious case suffers two successive MIP increases, as shown in Figure 2(b), we show that this is not the worst case. Considering that the MIP is $m$ in $(s-1)^{t h}$ round, so we have the values of MIP and $\delta_{i}^{x}$ shown in Table 2. Notice that the value of $\delta_{i}^{x}$ is captured 


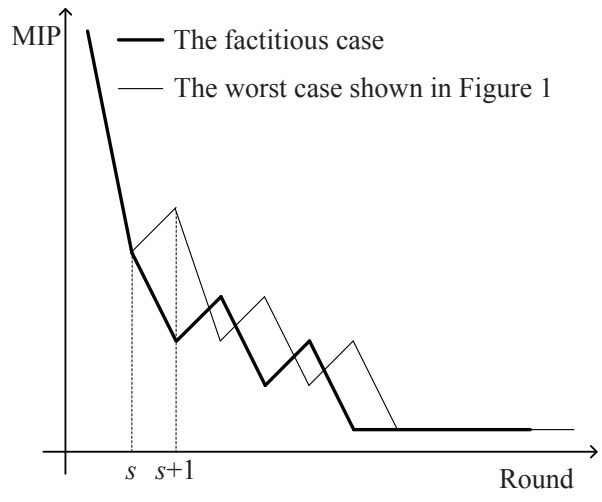

(a) Case 1: The two continuous MIP decreases.

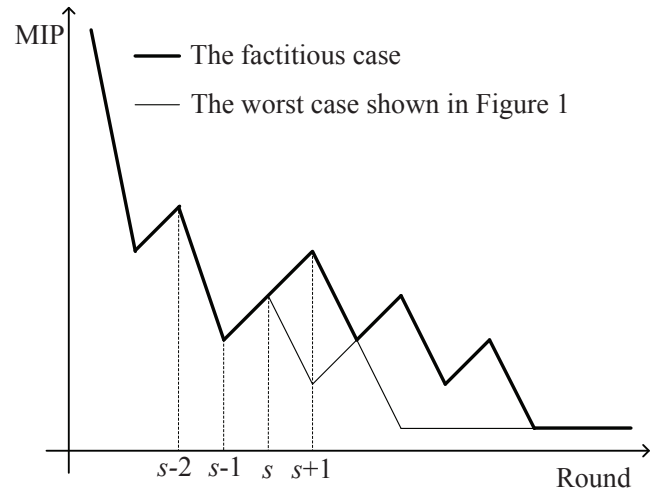

(b) Case 2: The two continuous MIP increases.

Fig. 2: The comparison of worst cases.

Table 2: The MIP variation of Figure 2(b).

\begin{tabular}{|c|c|c|}
\hline$x^{t h}$ Round & MIP & $\delta_{i}^{x}$ \\
\hline$x=s$ & $m+1$ & $m-1$ \\
\hline$x=s-1$ & $m$ & $2 m$ \\
\hline$x=s-2$ & $2 m+1$ & $2 m$ \\
\hline
\end{tabular}

before $a d_{i}$ proposes the bid price in $x^{\text {th }}$ round. It is clear that $a d_{i}$ can not update the bid price in $(s+1)^{t h}$ round because $\delta_{i}^{s+1}=m-1<m+1$. So, no two successive MIP increases will take place.

Combining above analyses together, the case illustrated in Figure 1 is the worst case of the convergent speed for the AIMD-MIP.

The following lemma shows the value of $h$. The label $m^{s}$ represents the MIP used in $x^{\text {th }}$ round.

Lemma 4. Suppose the MIP is first decreased in $(h+1)^{\text {th }}$ round, the lower bound of $h$ is $\sqrt[2]{\left(m^{1}\right)^{2}+2 \delta_{i}}-m^{1}$, where $\delta_{i}$ is the initial $A B P$ of $a d_{i}$.

Proof.In Definition 3, because the bid prices are updated in first $h$ rounds, we have:

$$
\sum_{s=1}^{h} m^{s} \geq \delta_{i} .
$$

The MIP is increased by one, so Eq. (6) can be rewritten as:

$$
\begin{aligned}
\frac{\left(m^{1}+m^{h}\right) h}{2} & \geq \delta_{i} \\
\frac{\left(m^{1}+m^{1}+h\right) h}{2} & \geq \delta_{i} \\
\frac{\left(2 m^{1}+h\right) h}{2} & \geq \delta_{i} .
\end{aligned}
$$

After solving Eq. (7), the lower bound of $h$ is derived.

$$
\begin{array}{r}
h \geq \frac{\sqrt[2]{4\left(m^{1}\right)^{2}+8 \delta_{i}}-2 m^{1}}{2} \\
h \geq \sqrt[2]{\left(m^{1}\right)^{2}+2 \delta_{i}}-m^{1}
\end{array}
$$

Then, the number of remainder rounds is shown in the following lemma.

Lemma 5.After the first MIP decrease, the NDSSA with AIMD-MIP requires at most $2\left\lceil\log \left(m^{1}+h-2\right)\right\rceil$ rounds to converge, where $h=\sqrt[2]{\left(m^{1}\right)^{2}+2 \delta_{i}}-m^{1}$.

Proof. Because the MIP is decreased in $(h+1)^{\text {th }}$ round, the value of $\delta_{i}^{h}$ is less than $m^{h}=m^{1}+h-1$. This implies $\delta_{i}^{h} \leq$ $m^{1}+h-2$. In the worst case, the MIP adaptation phrase exhausts the amount of bid raise $\left(m^{1}+h-2\right)$.

According to Lemma 3, the MIP variation in the MIP adaptation phrase consists of some pairs of the MIP decrease and MIP increase. In every MIP decrease, the value of $\delta_{i}^{s}$ is reduced to a half, so the convergence process requires at most $\left\lceil\log \left(m^{1}+h-2\right)\right\rceil$ rounds. Because every MIP decrease comes with an MIP increase, the NDSSA requires $2\left\lceil\log \left(m^{1}+h-2\right)\right\rceil$ rounds to converge after the first MIP decrease.

Theorem 2.Suppose $a d_{i}$ has the maximum $A B P \delta_{i}$, and the first MIP decrease takes place in $(h+1)^{\text {th }}$ round, i.e., $h=$ $\sqrt[2]{\left(m^{1}\right)^{2}+2 \delta_{i}}-m^{1}$. The convergent speed of the AIMDMIP in the worst case is:

$$
r^{a}=2\left(h+2\left\lceil\log \left(m^{1}+h-2\right)\right\rceil\right) .
$$

Proof.Combining Lemma 4 and 5, $a d_{i}$ requires at most $\left(h+2\left\lceil\log \left(m^{1}+h-2\right)\right\rceil\right)$ rounds to meet the stable outcome in the AIMD-MIP. According to Definition 3, the convergent speed of the AIMD-MIP is $r^{a}=2\left(h+2\left\lceil\log \left(m^{1}+h-2\right)\right\rceil\right)$. 


\subsection{Comparison of Convergent Speed}

We analyze the essential condition that the fixed-MIP converges faster than the AIMD-MIP.

Theorem 3.Given the initial MIP $\mathrm{m}^{1}$. Suppose ad has maximum $A B P \delta_{i}$, and the first MIP decrease takes place in $(h+1)^{\text {th }}$ round, where $h=\sqrt[2]{\left(m^{1}\right)^{2}+2 \delta_{i}}-m^{1}$. The fixed-MIP converges faster than the AIMD-MIP when $h \geq r^{f}-2 \log \left(\delta_{i}\right)$, where $r^{f}$ and $r^{a}$ are the convergent speed of the fixed-MIP and the AIMD-MIP.

Proof.Assume that the fixed-MIP converges faster than the AIMD-MIP, i.e., $r^{f} \leq r^{a}$. Combining the results in Theorem 1 and 2, we have:

$$
\begin{aligned}
r^{f} & \leq r^{a} \\
& =h+2 \log \left(m^{1}+h-2\right)
\end{aligned}
$$

Because the initial ABP of $a d_{i}$ is higher than $m^{h}$, i.e., $\delta_{i} \geq$ $\left(m^{1}+h-2\right)$, the lower bound of the first MIP decrease of the AIMD-MIP is:

$$
\begin{gathered}
r^{f} \leq h+2 \log \left(\delta_{i}\right) \\
h \geq r^{f}-2 \log \left(\delta_{i}\right) .
\end{gathered}
$$

\section{Experiments}

\subsection{Environment Setup}

We referred to $[1,18]$ to build our simulations. The valuation settings are considered within 10 and 50 . The instances of advertisers' valuations and CTRs are generated by linear, exponentially decreasing, exponentially increasing, and random distributions. Moreover, we consider a special case that the GSP outputs a non-truthful result [4]. The settings are shown in Table 3, and the special cases of valuation and CTR are marked as number four.

Initial MIPs are considered from 1 to 50 with gap 1 to measure the performance of various MIP adjusting strategies because the maximum valuation is 50. Each advertiser adopts the utility-maximization strategy formulated in section 3 to compute the bid price in each round. To realize the diversity of bidding in the real world, we generate five instances of initial bid prices at random.

\subsection{SEP Revenue in Stable Outcomes}

We first evaluate the SEP revenue in stable outcomes. Because the SEP revenue depends on the given initial MIP and MIP adjusting strategies, we consider all combinations of the initial MIP and MIP adjusting

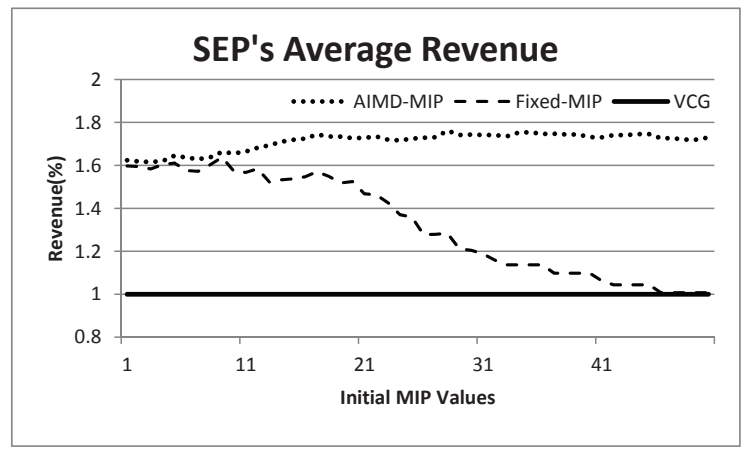

Fig. 3: SEP's revenue in the stable outcomes

strategies. Figure 3 shows the SEP revenue in average, and the base line is the VCG.

In the settings with small initial MIP $\left(m^{1} \leq 11\right)$, fixed-MIP performs similar to AIMD-MIP (1.63\% difference approximately). As the initial MIP is increased, the AIMD-MIP produces more and more SEP revenue, but the fixed-MIP obtains opposite results. After initial MIP 45, the SEP revenue in the AIMD-MIP is 1.7 times of that in the VCG, but the fixed-MIP performs very close to the VCG.

The SEP revenue in the fixed-MIP fully depends on the initial MIP. Inappropriate initial MIPs lead to low SEP revenue. On the other hand, initial MIP does not dramatically affect the SEP revenue in the AIMD-MIP. The revenue variance is bounded by $20 \%$ revenue obtained by the VCG. In summary, the AIMD-MIP outperforms the fixed-MIP in terms of the SEP revenue. The fixed-MIP with only appropriate initial MIP may generate the SEP revenue close to that derived by the AIMD-MIP.

\subsection{Overall Comparison for SEP Revenue in Stable Outcomes}

The overall comparison is a pairwise competition, and the results are shown in Figure 4. Each square includes three measurements $>,=$, and $<$ which represent the number of instances that the left mechanism is better than, equal to, and worse than the up-side mechanism, respectively. For example, in the center square, the fixed-MIP outputs more SEP revenue than the VCG in 5260 instances, ties in 98 instances, and less than the VCG in 892 instances.

AIMD-MIP is outstanding in 11134 instances (89.07\% approximately) and only worse than the fixed-MIP in 192 instances (1.54\% approximately). So, the SEP in the AIMD-MIP gains more revenue than in other mechanisms. The fixed-MIP produces more SEP revenue than other mechanisms in only 5452 instances (43.62\% approximately). In addition, the VCG outperforms the fixed-MIP in 892 instances $(14.27 \%$ 
Table 3: The valuation and CTR settings applied in our simulations.

\begin{tabular}{|c|ccccc|c|cccc|}
\hline \multicolumn{7}{|c|}{ Valuation Instances } & \multicolumn{5}{c|}{ CTR Instances } \\
\hline case \# & $a d_{1}$ & $a d_{2}$ & $a d_{3}$ & $a d_{4}$ & $a d_{5}$ & case \# & $s l_{1}$ & $s l_{2}$ & $s l_{3}$ & $s l_{4}$ \\
\hline 1 & 50 & 40 & 30 & 20 & 10 & 1 & 0.8 & 0.6 & 0.4 & 0.2 \\
\hline 2 & 50 & 34 & 22 & 14 & 10 & 2 & 0.8 & 0.376 & 0.164 & 0.053 \\
\hline 3 & 50 & 46 & 38 & 26 & 10 & 3 & 0.8 & 0.747 & 0.641 & 0.429 \\
\hline 4 & 50 & 45 & 30 & 25 & 10 & 4 & 0.8 & 0.76 & 0.4 & 0.38 \\
\hline 5 & 44.46 & 41.00 & 40.68 & 28.80 & 26.96 & 5 & 0.8 & 0.598 & 0.475 & 0.39 \\
\hline
\end{tabular}

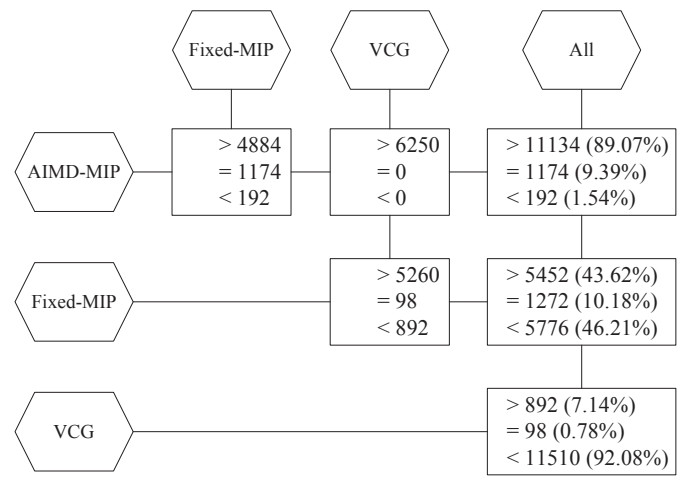

Fig. 4: The overall comparison of the SEP revenue for each mechanism.

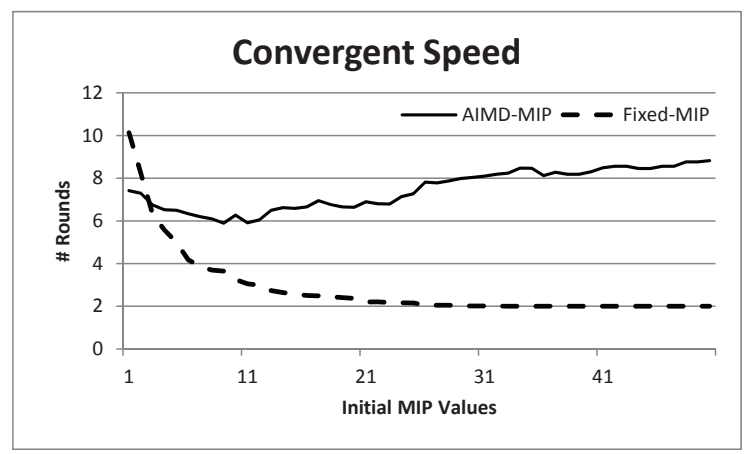

Fig. 5: The convergent speed comparison between the fixed-MIP and the AIMD-MIP.

approximately). Therefore, the SEP applying fixed-MIP must carefully determine the initial MIP.

\subsection{Convergent Speed}

The convergent speed is defined as the number of rounds that a mechanism requires to realize a stable outcome. The simulation result is shown in Figure 5, and smaller values indicate faster convergent speed.

For the simulations with initial MIP smaller than 11, the convergent speed of the fixed-MIP is enhanced dramatically as increasing the initial MIP settings.

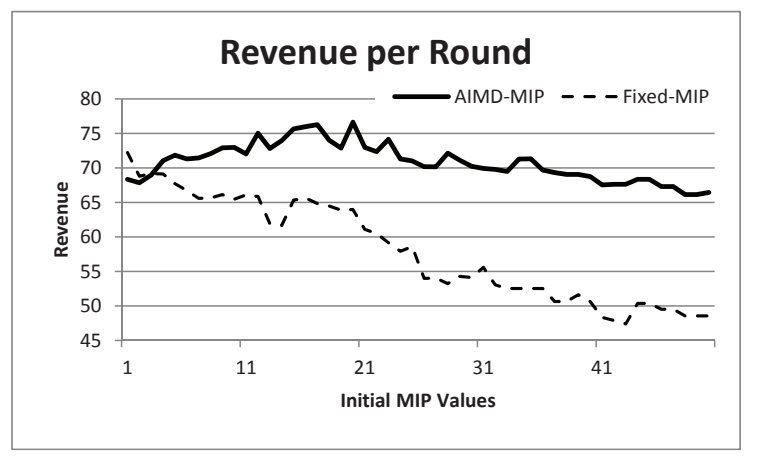

Fig. 6: The comparison result of Revenue-Per-Round.

Because the MIP is invariant throughout the auction, increasing the initial MIP leads to that advertisers can not update bid prices. For the simulations with initial MIP higher than 11, raising initial MIP settings slightly increases the convergent speed. Advertisers can not update bids if the MIP is higher than the ABP, so the mechanism requires fewer rounds to meet stable outcomes. This confirms the result derived in Theorem 1, where the convergent speed of the fixed-MIP is inversely proportional to the initial MIP.

The curve of the convergent speed of the AIMD-MIP is slightly dropped when $m^{1} \leq 11$ and then raised. Because the AIMD-MIP adjusts the MIP settings according to the bid variance, most advertisers can update bid prices to compete for the target slots. Therefore, the AIMD-MIP converges slower than the fixed-MIP in most instances, and the initial MIP settings do not affect the convergent speed too much.

The AIMD-MIP is an adaptive MIP adjusting strategy, and it requires more rounds to realize a stable outcome than the fixed-MIP in most instances. For popular keywords, the fixed-MIP with appropriate initial MIP is better than the AIMD-MIP. Because popular keywords arrives frequently, the SEP can learn the optimal MIP setting from auction experiences. The AIMD-MIP is suitable for rare keywords. Because rare keywords are issued infrequent, the SEP may not have enough experiences to study the appropriate initial MIP. 


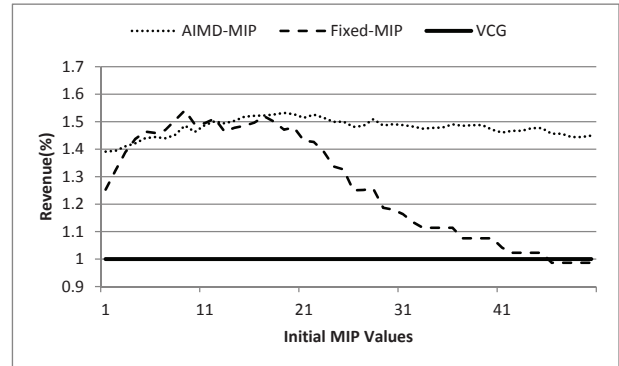

(a) Max: 50 rounds.

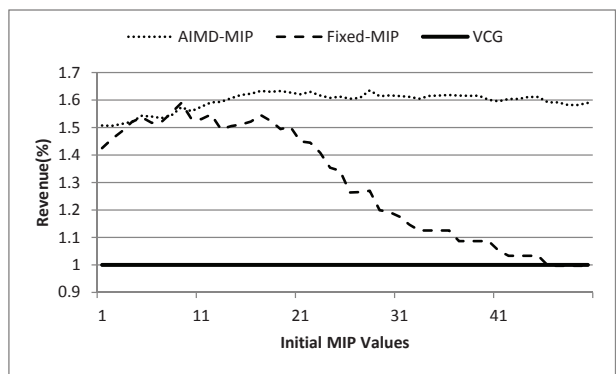

(c) Max: 100 rounds.

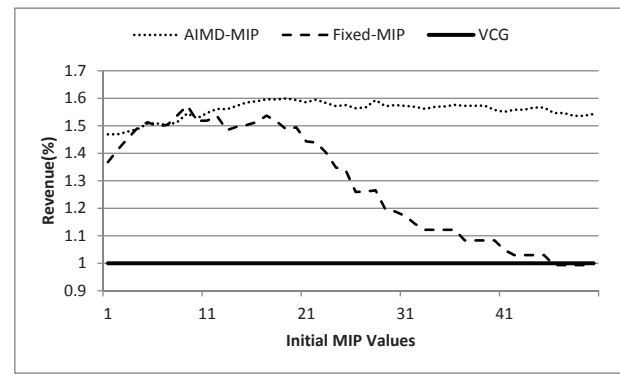

(b) Max: 75 rounds.

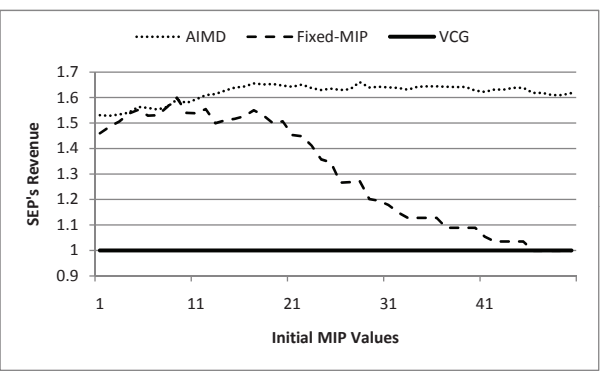

(d) Max: 125 rounds.

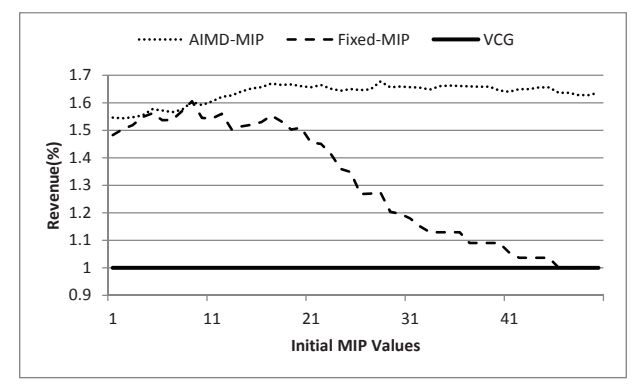

(e) Max: 150 rounds.

Fig. 7: SEP's long-term revenue comparison for the mechanisms with various maximum round restrictions.

\subsection{Revenue-Per-Round}

This simulation combines the SEP revenue in the stable outcome with the convergent speed. We focus on the SEP revenue obtained in the time period that the NDSSA does not converge. Suppose a mechanism $x$ requires $r^{x}$ rounds to converge, and the SEP receives the total revenue $f^{x}$ in this period. We use $f_{x} / r_{x}$ to indicate the revenue-per-round (RPR). Higher RPR values indicate that the SEP can gain more revenue in the unconvergent stage. Figure 6 shows the experiment result.

The RPR tendency of the fixed-MIP is getting worse as increasing the initial MIP. Advertisers can not update bid prices in the fixed-MIP with high initial MIP settings, so the bid value proposed in the first round determines the SEP revenue. Even though the number of rounds required to converge, i.e., the term $r^{x}$ in the RPR explanation, is reduced, the RPR of the fixed-MIP does not perform well in high initial MIP settings because most initial bid prices are low.

For the AIMD-MIP, the initial MIP setting does not affect the SEP revenue too much according to Figure 3. So, the RPR is proportional to the convergent speed. Because the variation of the convergent speed is small (the gap between the worst and best case is only three rounds), the RPR curve does not drop dramatically.

\subsection{Long-term Revenue}

The long-term revenue indicates the amount that the SEP gains within a time interval. We observe the SEP revenue by setting the maximum rounds as $50,75,100,125$, and 150. The results are shown in Figure 7, and the base line is VCG.

The curve tendencies of the fixed-MIP are similar in the results with various maximum rounds. The SEP 
revenue is increased in first nine rounds, and then continuously dropped. For the initial MIP larger than 45, the SEP in the VCG gains more revenue than in the fixed-MIP.

For the AIMD-MIP, various initial MIP settings slightly change the SEP long-term revenue. Increasing the maximum rounds results in more SEP revenue, and the raised amount is $10 \%$ comparing to the VCG. According to the result shown in section 6.2, the SEP revenue in stable outcomes of the AIMD-MIP is much higher than that in the VCG. Thus, the AIMD-MIP generates more long-term revenue as increasing the maximum rounds.

In Figure 7(a), the fixed-MIP outperforms the AIMD-MIP when the initial MIP is set as between three and twelve. However, as increasing the maximum rounds, the SEP gains less and less revenue. According to the results obtained in section 6.2, the AIMD-MIP outputs more SEP revenue in stable outcomes than the fixed-MIP, so increasing the maximum rounds is disadvantageous for the fixed-MIP. Thus, we conclude following properties for the long-term revenue: the fixed-MIP with appropriate initial MIP setting is suitable for rare keywords (fewer rounds a day) while the AIMD-MIP is outstanding in popular keywords (more rounds a day).

\section{Conclusion}

In this paper, we developed the NDSSA to reduce the cost of computing an auction result. To determine the MIP setting for maximizing the SEP revenue, we propose the fixed-MIP and the AIMD-MIP. The convergent speed is analyzed, and the essential conditions that the fixed-MIP converges faster than the AIMD-MIP is derived. We conclude the following properties from our simulations. For all the SEP revenue considerations, AIMD-MIP is outstanding in popular keywords, and rare keywords for the fixed-MIP. Opposite results are obtained for the convergent speed.

We currently consider that each advertiser adopts the utility-maximization strategy to compute bid values. This is not sufficient to formulate the real world bidding behavior. So, we will apply the data mining techniques $[20,21,22]$ to find out the practice bidding strategies. Then, we will evaluate the performance of the NDSSA for the advertisers with the obtained bidding strategies.

\section{References}

[1] M. Cary, A. Das, B. Edelman, I. Giotis, K. Heimerl, A. R. Karlin, C. Mathieu, and M. Schwarz, Greedy Bidding Strategies for Keyword Auctions, Proceedings of the 8th ACM Conference on Electronic Commerce (EC-07), 262-271 (2007).

[2] T. M. Bu, X. Deng, and Q. Qi, Forward looking Nash equilibrium for keyword auction, Information Processing Letters, 105, 41-46 (2008).
[3] Y. Zhou and R. Lukose, Vindictive Bidding in Keyword Auctions, Proceedings of 9th international conference on Electronic Commerce (ICEC07), 141-146 (2007).

[4] B. Edelman, M. Ostrovsky, and M. Schwarz, "Internet Advertising and the Generalized Second Price Auction: Selling Billions of Dollars Worth of Keywords," American Economic Review, 97, 242-259 (2007).

[5] M. Babaioff and T. Roughgarden, Equilibrium Efficiency and Price Complexity in Sponsored Search Auctions," Workshop on Ad Auctions, (2010).

[6] B. Edelman and M. Ostrovsky, Strategic Bidder Behavior in Sponsored Search Auctions," Decision Support Systems, 43, 192-198 (2007).

[7] D-M Chiu and R. Jain, Analysis of the Increase and Decrease Algorithms for Congestion Avoidance in Computer Networks, Computer Networks and ISDN Systems, 17, 1-14 (1989).

[8] D. Bansal and H. Balakrishnan, Binomial Congestion Control Algorithms, IEEE Infocom, (2001).

[9] D. E. Comer, Internetworking with TCP/IP, Prentice Hall Press, (2006).

[10] Carr, Paul G., Investigation of Bid Price Competition Measured through Prebid Project Estimates, Actual Bid Prices, and Number of Bidders, Prentice Hall Press, (2006).

[11] H. R. Varian, "Position Auction," International Journal of Industrial Organization, 25, 1163-1178 (2007).

[12] Y. Narahari, D. Garg, R. Narayanam, and H. Prakash, "Game Theoretic Problems in Network Economics and Mechanism Design Solutions,” Springer, (2009).

[13] V. Krishna, Auction Theory, Academic Press, (2002).

[14] J. Zhang, H. C. Lau, and J. Shen, Setting discrete bid levels adaptively in repeated auctions, Proceedings of the 11th International Conference on Electronic Commerce, 195-204 (2009).

[15] A. Rogers, E. David, J. SchiR, S. Kraus, and N. R. Jennings, Learning environment parameters for the design of optimal English auctions with discrete bid levels, Proceedings of AAMAS, AMEC, (2005).

[16] E. David, A. Rogers, N. R. Jennings, J. SchiR, S. Kraus, and M. H. Rothkopf, Optimal design of English auctions with discrete bid levels, ACM Transaction on Internet Technology (TOIT), 7, 1-34 (2007).

[17] M. H. Rothkopf and R. Harstad, On the role of discrete bid levels in oral auctions, European Journal of Operational Research, 74, 572-581 (1994).

[18] M. Naldi, G. DAcquisto, and G. F. Italiano, The Value of Location in Keyword Auctions, Electronic Commerce Research and Applications, 9, 160-170 (2010).

[19] Michael Zhang and Juan Feng, Cyclical Bid Adjustments in Search-Engine Advertising, Management Science, 57, 17031719 (2011).

[20] Bringer J. and Chabanne H. Embedding edit distance to enable private keyword search. Human-centric Computing and Information Sciences, 2, (2012).

[21] Oommen B. J., Yazidi A. and Granmo O. An Adaptive Approach to Learning the Preferences of Users in a Social Network Using Weak Estimators. Journal of Information Processing Systems, 8, 191-212 (2012).

[22] M. Thangamani and P. Thangaraj, Fuzzy Ontology for Distributed Document Clustering based on Genetic Algorithm, Applied Mathematics \& Information Sciences, 7, 1563-1574 (2013). 


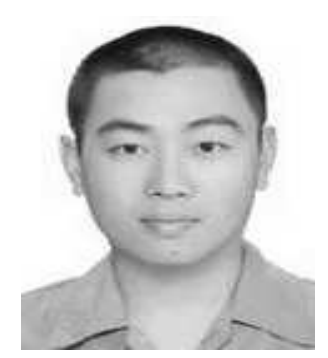

\section{Chen-Kun Tsung} received B.S. and M.S. degrees in the Department of Computer Science and Information Engineering from Da-Yeh University, Taiwan, ROC in 2004 and 2006, respectively. $\mathrm{He}$ is currently working toward a $\mathrm{PhD}$ degree in the Institute of Computer Science and Information Engineering at National Chung Cheng University, Taiwan, ROC. His research areas include game theory, mechanism design, and economics computation.

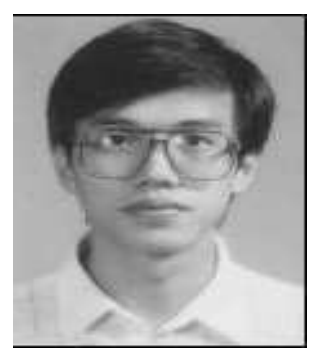

Hann-Jang Ho received B.S. and M.S. degrees in Electronic Engineering from National Taiwan University of Science and Technology in 1988 and 1990 respectively, and the Ph.D. degree in Computer Science and Information Engineering from National Chung Cheng University, Taiwan, ROC, in 2003. He is currently an Associate Professor in the Department of Applied Digital Media, WuFeng University, Taiwan, ROC.

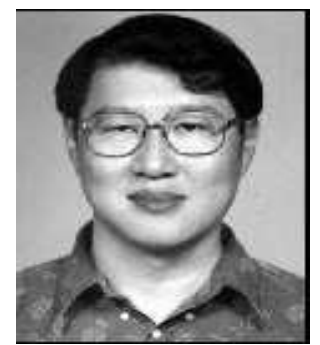

Sing-Ling Lee received a BS degree in Mathematical Science from National Central University, Taiwan, ROC in 1976 and the M.S. and Ph.D. degrees in computer science from University of Texas at Dallas, USA, in 1981 and 1984, respectively. He is currently a Professor in the Department of Computer Science and Information Engineering, National Chung Cheng University, Taiwan, ROC. His research areas include algorithms, mobile computation, optical computation, internet computing, machine learning, and economics computation. Dr. Lee is a member of ACM and IEEE. 